\title{
Modélisation et simulation des écoulements en situations ind ustrielles Exposé de synthèse
}

\author{
Numerical prediction of turbulent flows in engineering \\ Synthesis report
}

\author{
Ha Minh Hieu
}

Institut de mécanique des fluides de Toulouse

\begin{abstract}
L'objet de cette contribution est de faire un bilan synthétique des différentes approches statistiques utilisées jusqu'ici pour la prédétermination numérique des écoulements turbulents complexes généralement rencontrés par l'Ingénieur. Ainsi. des modèles de turbulence à concept de viscosité tourbillonnaire ou des modèles au second ordre, et leurs incidences sur les aspects numériques, sont présentés. En rappelant les difficultés à surmonter actuellement, on laisse entrevoir des possibilités de simulation numérique des écoulements industriels dans un avenir proche.
\end{abstract}

The objective of this review is to discuss about several statistical approaches used to predict numerically complex turbulent flows in engineering. Both physical concepts and numerical aspects are discussed. Are also presented some research trends in turbulent modelling and some perspectives in the next future, for engineering problems.

\section{Introduction}

L'un des vieux rêves de l'ingénieur est la possibilité de prédéterminer les phénomènes physiques qu'il étudie sans recourir à l'expérience physique. Pour satisfaire à cette demande, les chercheurs ont essayé sans cesse de mettre en "équations » les phénomènes physiques, de résoudre ces équations et de comparer les résultats de calcul obtenus aux données expérimentales. Lorsque la comparaison est satisfaisante, ces équations reçoivent alors le label de "modèles mathématiques".

Ainsi, durant les 30 dernières années, les instruments de travail de l'ingénieur ont subi d'énormes évolutions. A la place des catalogues contenant des formules toutes faites et des abaques toutes tracées, l'ingénieur ressent de plus en plus le besoin de "revenir aux sources" en se tournant vers les Principes fondamentaux de la physique, vers les équations qui en découlent mais aussi vers les méthodes de résolution de ces dernières.
Le premier «choc pétrolier» de 1973 a modifié profondément la méthodologie de l'ingénieur. Il ne peut plus de contenter des méthodes globales et de ces "théories des coefficients", mais il doit affronter de plus en plus des problèmes d'optimisation poussée des systèmes, opération largement épaulée par des ordinateurs de plus en plus puissants et de prix de revient (par opération) de moins en moins élevés.

La mécanique des fluides est une branche de la physique qui, depuis toujours de trouve être le champ d'application des progrès de la résolution numérique des équations aux dérivées partielles, et donc de l'utilisation des gros ordinateurs. Cela est due à un fait : la mécanique des fluides est riche en non-linéarité.

Cette non-linéarité omniprésente n'est pas sans conséquence importante sur la plupart des problèmes traités, aussi bien sur le plan physique que numérique. Même dans des fluides courants tels que l'air et l'eau, où la loi de comportement est linéaire (loi newtonnienne), la 
non-linéarité convective pose des problèmes d'existence et d'unicité de la solution pour le modèle résultant, c'est-à-dire pour le modèle classique de Navier-Stokes. A cette non-linéarité convective, il convient d'en ajouter trois autres: i) la première peut provenir des variations de densité, qui renforcent le couplage entre les équations de transport, toujours au niveau de la convection, ii) la seconde venant des termes sources importants par exemple les effets de flottabilité et iii) la troisième, qui n'est pas la moindre, tire son origine de la loi de comportement. A ce titre, des lois rhéologiques non linéaires, si importantes en situations industrielles, peuvent avoir un rôle important dans la stabilité des écoulements. De ces faits, bien peu d'écoulements restent en régime laminaire longtemps après leur démarrage : ils deviennent alors turbulents avec toutes les conséquences que cela comporte tant sur le plan structurel que sur celui des mécanismes de transfert.

Quoiqu'il en soit, les écoulements turbulents sont beaucoup plus complexes que leurs homologues laminaires, à cause de la présence d'échelles caractéristiques multiples comme nous le verrons plus tard. C'est pour cette raison que les écoulements turbulents se prêtent difficilement aux méthodes de résolution analytiques et qu'une résolution numérique complète se révèle alors souvent nécessaire. Mais ceci n'est pas la seule cause de l"utilisation du numérique en mécanique des fluides.

Les années 70 ont connu un extraordinaire essor du développement des méthodes de prédétermination par voie numérique des écoulements turbulents. Ce fait est dû à une heureuse conjonction de maturité de trois disciplines: i) modélisation mathématique des phénomènes physiques d'écoulements et, plus particulièrement, de la turbulence; ii) analyse numérique et ses applications à la résolution des équations aux dérivées partielles non linéaires, et iii) avènement des calculateurs à grandes capacités de mémoires et à grandes vitesses de calcul.

L'ampleur des progrès réalisés peut être appréciée lorsqu'on compare des résultats obtenus entre le premier congrès de Stanford en 1969 [1] et le second du même nom en 1981 [2]. A de simples écoulements cisaillés minces, on est passé rapidement aux écoulements complexes incluant souvent de forts décollements avec recirculations, des interactions choc/couche-limite, de fortes variations de densité, des situations tridimensionnelles, en milieux hétérogènes et mêmes en milieux réactifs...

Quelle que soit la configuration d'écoulement étudiée, les travaux réalisés dans les années 70 s'inscrivent bien souvent dans un cadre très classique : situations isovolumes et mouvements stationnaires en moyenne. En fait, les modèles de turbulence, développés dans les années 70 , ont donné des satisfactions dans la prédétermination numérique des écoulements cisaillés minces (jets, sillage, zones de mélange...) et, généralement, dans les parties pleinement développées des écoulements où l'on observe des propriétés auto-similaires des grandeurs moyennes et turbulentes. La prédétermination théorique semble être moins satisfaisante dans des zones de transition et de création de la turbulence. De même, si certaines prédéterminations numériques des écoulements décollés, avec d'importantes parties de recirculation, semblent être convenables dans des zones de mélange, les prévisions théoriques des transferts pariétaux (quantité de mouvement, de masse et de chaleur) sont encore perfectibles.
Les années 80 sont davantage marquées par des efforts de modélisation des effets complexes de la turbulence. Sous le vocable " complexe ", on entend les effets non pris en compte jusqu'alors: effets de fortes courbures, effets de forces de volume, effets de l'instationnarité des grandeurs moyennes, effets de densité variable ou d'inhomogénéité massique, etc.

A cause des exigences imposées par une grande diversité de problèmes industriels, un des défis à relever durant cette décade dans le domaine de la modélisation de la turbulence, est la prise en compte correcte des effets complexes précédemment cités.

Pour souligner ces efforts par rapport aux situations actuelles, il convient de revenir sur des acquis obtenus dans le domaine des écoulements isovolumes.

\section{Ecoulements isovolumes : modèle de base}

Dans le cadre des milieux continus, les équations de conservation de masse et de quantité de mouvement, et la loi de comportement newtonienne linéaire fournissent le modèle de base de Navier-Stokes:

$$
\begin{gathered}
\frac{\partial U_{k}}{\partial x_{k}}=0 \\
\left(\frac{\partial U_{i}}{\partial t}+U_{k} \frac{\partial U_{i}}{\partial x_{k}}\right)=-\frac{\partial P}{\partial x_{i}}+\nu \frac{\partial^{2} U_{i}}{\partial x_{k} \partial x_{k}}
\end{gathered}
$$

L'évolution isovolume de l'écoulement a permis au problème dynamique d'être indépendant du problème énergétique, l'équation gouvernant le premier principe de la thermodynamique est donc délaissée pour le moment.

La non-linéarité de l'équation (2) la rend sensible aux perturbations éventuelles introduites par toute méthode de résolution par approximation, au moins à partir d'un certain seuil du nombre de Reynolds caractéristique; ce paramètre étant un indicateur du degré de non-linéarité de l'écoulement. Bien que la correspondance entre ces « perturbations numériques" et les "perturbations physiques" (irrégularités des conditions aux limites par exemple) ne soit pas complètement établie sur le plan mathématique, on observe cependant que les solutions numériques obtenues contiennent bien des caractéristiques analogues à celles des écoulements turbulents [3].

La remarque précédente permet donc de renforcer le postulat suivant: puisque les échelles caractéristiques de la turbulence sont encore largement supra-moléculaires, le concept de la particule fluide n'est pas remis en cause dans ce type d'écoulement. Par suite, le modèle de Navier-Stokes reste donc représentatif des mouvements instantannés en régime turbulent. Ce postulat conduit donc tout naturellement à une première approche (classement logique mais non chronologique): la simulation numérique directe de la turbulence, sans aucune modélisation supplémentaire. Les caractères tridimensionnel et instationnaire de la turbulence et la présence d'un large spectre de tourbillons conduisent d'une part à des calculs très coûteux sur supercalculateurs, et d'autre part à des écoulements à des nombres de Reynolds encore modestes par rapport aux situations industrielles. Bien que des 
résultats numériques très intéressants soient obtenus pour des écoulements académiques tels que : couche limite sans gradient de pression [4], ou avec relaminarisation [5], couche limite sur une paroi courbée [6], ou dans un canal [7], cette voie n'est pas encore très bien adaptée aux problèmes industriels essentiellement pour des raisons de coûts excessifs (*). De ce fait, et pour l'instant, on peut considérer cette démarche comme un moyen d'acquérir un grand nombre de "données expérimentales » (expériences numériques) extrêmement précieuses pour la modélisation mathématique des aspects physiques complexes précités. Nous ne discuterons donc pas cette approche dans cet exposé.

A partir du moment où l'on renonce à suivre en détail le mouvement de toutes les structures présentes dans un écoulement turbulent, il faut bien faire un choix dans la représentativité des grandeurs caractéristiques de cet écoulement.

L'idée n'est pas nouvelle: devant la complexité des mouvements turbulents étudiés, déjà Reynolds (1878) a proposé de décomposer le mouvement instantanné en une partie moyenne et une partie fluctuante, arguant du fait que pour des problèmes pratiques d'Ingénieur, la connaissance des grandeurs moyennes est largement suffisante. Il a proposé alors un traitement statistique des équations avant toute résolution numérique, ce qui est tout à fait à l'opposé de la simulation directe de la turbulence où le traitement statistique n'intervient qu'à la fin, sur l'ensemble des réalisations numériques.

L'approche statistique appelle deux remarques: i) d'une part, appliquée à un processus non-linéaire, elle conduit toujours à un accroissement du nombre d'inconnues, ce qui pose évidemment un problème de fermeture; ii) d'autre part, pour permettre une confrontation expérimentale, on assimile bien souvent les moyennes statistiques d'ensemble aux moyennes temporelles. Cela n'est possible que dans un cadre bien précis de stationnarité et d'ergodicité. De ce fait, bien des écoulements industriels réellement instationnaires se trouvent exclus de cette approche. L'instationnarité n'a pu être incluse dans la modélisation statistique classique car celle-ci s'inscrit dans un cadre bien précis: l'équilibre spectral au sens de Kolmogorov. En d'autres termes, tout le spectre d'énergie des fluctuations est représenté par sa valeur à la fréquence nulle, excluant toute possibilité d'échange énergétique avec l'extérieur, à d'autres fréquences.

Pour remédier à cela, une troisième approche s'est dégagée depuis les années 70; elle cherche à combiner les deux précédentes: une simulation directe tridimensionnelle et instationnaire de toutes les structures de taille supérieure à une certaine dimension imposée par un « filtre » et une modélisation statistique simplifiée sur les effets des plus petites structures. Cette voie, dite de simulation des macro-échelles a déjà donné des résultats

(*) Cette position doit être prise avec réserve : dans un passé récent nous avions pensé que l'approche des milieux continus est la seule raisonnable pour des situations industrielles et que l'approche moléculaire est hors de portée des problèmes d'Ingénieur. Les remarquables résultats obtenus récemment par d'Humières, Lallemand, Pommeau [8] pour un gaz de réseau ont montré que dans un avenir relativement proche, cette démarche serait disponible pour des configurations d'écoulement plus complexes. très intéressants dans des écoulements académiques simples. L'extension de telles méthodes aux écoulements complexes en situations réelles mérite encore des approfondissements sur le plan aussi bien de la méthodologie que de la modélisation théorique.

Nous ne portons ici notre discussion que dans le cadre de la modélisation statistique classique et n'interviendrons que très ponctuellement dans les deux autres démarches.

\section{Aspects de la modélisation dans l'approche statistique}

Ainsi, dans un traitement statistique conventionnel, on obtient à la place des équations de Navier-Stokes, le système des équations de Reynolds:

$$
\begin{gathered}
\frac{\partial \bar{U}_{k}}{\partial x_{k}}=0 \\
\frac{\partial \bar{U}_{i}}{\partial t}+\bar{U}_{k} \frac{\partial}{\partial x_{k}} \bar{U}_{i}+\frac{\partial}{\partial x_{k}} \overline{u_{i} u_{k}}=-\frac{1}{\rho} \frac{\partial \bar{P}}{\partial x_{i}}+v \frac{\partial^{2} \bar{U}_{i}}{\partial x_{k} \partial x_{k}}
\end{gathered}
$$

Bien que l'origine des corrélations d'ordre deux soit la non-linéarité convective, le fait de les transformer en contraintes turbulentes $\overline{u_{i} u_{k}}$ et de les regrouper aux autres contraintes existantes préconditionne déjà une certaine modélisation. En effet, la première idée que l'on peut avoir est d'emprunter la même démarche antérieurement adoptée lors de la formulation de la loi newtonienne de comportement :

$$
-\rho \overline{u_{i} u_{k}}=\mu_{i}\left(\frac{\partial \bar{U}_{i}}{\partial x_{k}}+\frac{\partial \bar{U}_{k}}{\partial x_{i}}\right)-\frac{2}{3} \delta_{i k} \rho \bar{k}
$$

Le terme isotrope que l'on a ajouté ici, à l'origine pour assurer la validité de l'égalité (5) dans le cas de la contraction des indices, en fait attribue à l'énergie cinétique de la turbulence: $\rho \bar{k}=0,5 \rho \overline{u_{i} u_{k}}$ le rôle d'une pression due aux mouvements d'agitation.

Toutes les démarches qui s'appuyent sur la relation (5), dite de Boussinesq, appartiennent à la classe des modèles au concept de viscosité turbulente. Une autre alternative de modélisation qui ne recourt pas à ce concept est la modélisation au second ordre qui consiste à considérer les contraintes turbulentes (moments du second ordre) comme des grandeurs transportables susceptibles d'avoir une histoire propre d'évolution.

\subsection{Modélisation à concept de viscosité turbulente}

La loi de comportement proposée (5) montre que le problème de modélisation se ramène maintenant à la détermination d'une viscosité turbulente $\mu_{t}$, c'est-à-dire d'un couple d'échelles caractéristiques de vitesse et de longueur. Mais contrairement à la viscosité moléculaire où ces échelles sont empruntées au mouvement d'agitation des molécules (ce qui confère à cette même viscosité le rôle d'une propriété physique intrinsèque au fluide), la viscosité turbulente doit être caractérisée par des échelles représentatives du mouvement turbulent lui-même. Ce dernier mouvement est caractérisé par des échelles largement supramoléculaires (donc peu d'interaction avec le mouvement des molécules) qui sont par contre en forte 
connexion avec le mouvement moyen (au sens statistique): la viscosité turbulente est donc une propriété de l'écoulement.

Mais quelles échelles de vitesse et de longueur peut-on choisir pour représenter correctement la viscosité turbulente ? La remarque précédente montre que dune part ces échelles ne sont pas indépendantes du mouvement macroscopique moyen, et d'autre part, elles doivent êtgre évolutives dans l'espace et éventuellement dans le temps: la viscosité turbulente n'est donc pas constante dans le domaine d'écoulement.

L'examen des bilans énergétiques entre les différents mouvements a montré que le mouvement turbulent voit son énergie alimentée par le mouvement moyen lui-même. Ce mécanisme de production se fait à travers une classe particulière de tourbillons (dits tourbillons porteurs d'énergie) dont la fréquence caractéristique est voisine de celle du mouvement moyen (c'est cela qui a favorisé le transfert de l'énergie). Ainsi, l'échelle de vitesse $u$ et l'échelle de longueur $\ell$ caractéristiques du mouvement turbulent sont telles que :

$$
\frac{u}{\ell} \sim \frac{U_{0}}{L_{13}}
$$

C'est sur cette idée d'accord fréquentiel que sont fondés les schémas de fermeture de type longueur de mélange, dont le plus célèbre est celui de Prandlt (1925) :

$$
u \sim \ell\left(\frac{\partial \bar{U}_{i}}{\partial x_{k}}+\frac{\partial \bar{U}_{k}}{\partial x_{i}}\right)
$$

L'échelle de longueur $\ell$ qui reste à déterminer sera alors choisie empiriquement de telle manière qu'elle puisse représenter les gros tourbillons qui assurent la diffusion transversale des grandeurs moyennes. C'est pour cette raison que dans la plupart des écoulements académiques où l'on peut déterminer de façon expérimentale des lois d'évolution de cette échelle $\ell$, on constate qu'elle est proportionnelle à l'étendue transversale de l'écoulement en question.

Malgré la simplicité de mise en œuvre de ces modèles à zéro-équation (c'est-à-dire aucune équation de transport supplémentaire à ajouter au système initial des équations de conservation), ceux-ci ont donné d'excellents résultats dans des écoulements cisaillés minces où l'on peut admettre d'une part une hypothèse d'équilibre (production = dissipation) dans les deux équations de transport de l'énergie cinétique de la turbulence $\bar{k}$ et du cisaillement turbulent $u v$, et d'autre part une certaine proportionnalité entre ces deux grandeurs (hypothèse de Bradshaw). Les lois de Prandlt [9], de Cebeci-Smith-Mosinskis [10], de Balwin-Lomax [11], pour ne citer que les plus utilisées, qui ne diffèrent que sur la détermination d'une échelle transversale, ont donné des résultats satisfaisants dans les cas précédemment cités. Cependant tous ces modèles à zéroéquation souffrent d'un manque d'universalité : les lois d'échelle sont trop empiriques et supposent une connaissance au préalable de l'écoulement à calculer, ce qui ne correspond pas à la démarche de l'ingénieur appelé à concevoir des systèmes nouveaux.

L'étude spectrale des fluctuations de vitesse montre qu'une grande partie de l'énergie du mouvement fluctuant est contenue dans les gros tourbillons (mais pas les plus gros). Il est donc naturel de penser que l'échelle de vitesse caractéristique du mouvement fluctuant est directement reliée à l'énergie cinétique de la turbulence.

L'énergie cinétique de la turbulence sera alors calculée à partir d'une équation de transport, directement déduite des équations de Navier-Stokes. L'équation exacte ainsi établis contient comme précédemment de nouvelles corrélations inconnues: le problème de fermeture se pose à nouveau. La modélisation de cette équation est désormais une affaire classique, elle est basé sur les mécanismes physiques de la cascade énergétique de Kolmogorov et des étirements des tourbillons, tout cela dans un cadre des écoulements franchement turbulents (grands nombres de Reynolds de la turbulence). L'équation de transport modélisée de l'énergie cinétique de la turbulence s'écrit alors :

$$
\begin{aligned}
\frac{\partial}{\partial t} \bar{k} & +\bar{U}_{k} \frac{\partial \bar{k}}{\partial x_{k}}=-\overline{u_{i} u_{k}} \frac{\partial \bar{U}_{i}}{\partial x_{k}} \\
& +\frac{\partial}{\partial x_{k}}\left(\left(v+\frac{v_{i}}{\sigma_{h}}\right) \frac{\partial \bar{k}}{\partial x_{k}}\right)-\bar{\varepsilon}+W_{k}
\end{aligned}
$$

où $\bar{\varepsilon}$ désigne le taux de dissipation de l'énergie cinétique de la turbulence et $W_{k}$ des termes correctifs pour des zones visqueuses très proches des parois. De même, on a :

$$
v_{i}=C_{k} \sqrt{k} \cdot \ell \quad \text { et } \quad \bar{\varepsilon}=\frac{\bar{k}^{2}}{\ell}
$$

l'échelle de longueur $\ell$ sera prescrite algébriquement (et empiriquement) comme dans des modèles précédents à zéro-équation. Les constantes $C_{k}$ et $\sigma_{k}$ sont déterminées expérimentalement à partir des configurations d'écoulement particulières. Ces modèles, dits à une équation (de transport supplémentaire) ont fourni des résultats très acceptables dans des écoulements cisaillés simples.

Leur principale faiblesse réside toujours dans la difficulté de trouver a priori une loi algébrique correcte pour l'échelle de longueur $\ell$ dans des écoulements industriels complexes.

La question suivante se pose alors naturellement: peut-on établir une équation de transport pour l'échelle $\ell$, nous dispensant ainsi de la démarche trop empirique jusqu'ici ?

A l'exception de la détermination indirecte par Rotta [12] d'une équation de transport du produit $k_{1}$, à partir de l'évolution des corrélations spatio-temporelles, il n'est pas possible d'obtenir, de façon exacte, une équation relative à l'échelle $\ell$.

Par contre, il est possible d'établir, de façon exacte à partir des équations de Navier-Stokes, une équation de transport pour le taux de dissipation $\bar{\varepsilon}$, qui nécessite également une modélisation. En utilisant la relation (10) on peut alors décrire l'évolution de l'échelle de longueur caractéristique. La modélisation est réalisée à partir d'une analyse physique de l'enstrophie (intensité moyenne du rotationnel fluctuant). Ainsi, dans les modèles à deux équations (supplémentaires), à l'équation de l'énergie cinétique de la turbulence (9), s'ajoute l'équation modélisée gouvernant l'évolution du taux de dissipation :

$$
\begin{aligned}
\frac{\partial \bar{\varepsilon}}{\partial t}+\bar{U}_{k} \frac{\partial}{\partial x_{k}} \bar{\varepsilon}=C_{i} v_{i} \frac{\bar{\varepsilon}}{\bar{k}} \frac{\partial \bar{U}_{i}}{\partial x_{k}}\left(\frac{\partial \bar{U}_{i}}{\partial x_{k}}+\frac{\partial \bar{U}_{k}}{\partial x_{i}}\right) \\
-C_{2} \frac{\bar{\varepsilon}^{2}}{\bar{k}}+\frac{\partial}{\partial x_{k}}\left[\left(v+\frac{v_{i}}{\sigma_{i}}\right)\right] \frac{\partial \bar{\varepsilon}}{\partial x_{k}}+W_{i}
\end{aligned}
$$


où le terme $W_{k}$ représente les effets visqueux dans la proximité des parois solides. Les constantes $C_{1}=1,44, C_{2}$ $=1,92, \sigma_{k}=1,0$ et $\sigma_{t:}=1,30$, supposées universelles, ont été obtenues expérimentalement.

Ce modèle à deux équations, universellement connu sous le nom du modèle $(k, \varepsilon)$, est développé à l’origine par Jones et Launder en 1972 [13]. A l'heure actuelle, ce modèle très populaire, est vraisemblablement le plus utilisé, non seulement dans des études à caractère théorique, mais également dans des problèmes pratiques d'ingénieur. Même si, dans le plupart des cas, ce modèle a fourni des résultats satisfaisants, il est toutefois recommandé de ne pas perdre de vue les suivants:

Sur le plan fondamental, accepter le concept de viscosité turbulente (5) revient à conférer aux contraintes turbulentes, grandeurs ayant une origine convective non-linéaire, un caractère diffusif de type gradient donc de nature linéaire. Par suite, il faut s'attendre à ce que les effets de la turbulence stabilisent les mécanismes instationnaires issus de la non-linéarité des équations de Reynolds.

L'utilisation d'une viscosité turbulente représentée par le rapport $\left(\bar{k}^{2} / \bar{\varepsilon}\right)$ conduit à une valeur de diffusivité quasi-constante dans une grande partie du domaine des écoulements. En effet, les termes sources (production et destruction) de l'énergie cinétique d'une part et du taux de dissipation turbulente d'autre part sont quasi-proportionnels entre eux. De ce fait, une simulation numérique avec viscosité constante (donc faux régime laminaire) conduit généralement à des résultats analogues (sauf dans la zone des sous-couches visqueuses) mais à des coûts de calcul bien moins élevés. Par ailleurs, cette viscosité turbulente en $\left(\bar{k}^{2} / \bar{\varepsilon}\right)$ est également à l'origine de la difficulté de bien calculer la diffusivité de la quantité de mouvement longitudinale dans des écoulements à recirculation.

Une autre faiblesse du concept de viscosité turbulente est la représentation des trois contraintes turbulentes normales par leur somme, ce qui revient à admettre une évolution semblable de ces trois composantes, même si l'anisotropie turbulente de l'écoulement peut être contenue dans cette modélisation. De ce fait, dans des écoulements où les trois contraintes normales varient de façons très différentes (zones internes près des parois, chocs, détentes, distorsions rapides, zones à recirculation...), les modèles à concept de viscosité, même à deux équations, ne sont pas toujours très satisfaisants [14].

Le concept de viscosité turbulente a aussi des conséquences importantes sur le plan de la résolution numérique des équations. En effet, l'adoption d'une viscosité turbulente, par définition beaucoup plus importante que la viscosité moléculaire, conduit en réalité à des nombres de Reynolds locaux (rapport entre la convection et la diffusion locales) relativement faibles. Cela a permis à des schémas numériques, déjà bien opérationnels en écoulements aux nombres de Reynolds peu élevés, d'être étendus sans trop de difficulté aux écoulements turbulents, bien que le nombre de Reynolds nominal de l'écoulement soit bien plus élevé.

Enfin, dans la plupart des calculs réalisés jusqu'ici, bien peu de schémas de type complètement implicites ont été utilisés pour la résolution des équations (9) et (11). De ce fait, dans la résolution numérique pratique, mêmes les méthodes baptisées implicites connaissent en réalité une limitation en pas temporel pour des approches instationnaires. De même, la non-linéarité des termes sources nécessite presque toujours une linéarisation temporelle locale.

Les modèles à deux équations ont fait l'objet de très nombreuses applications, même en situations complexes. Dans une excellente revue [15], Rodi a présenté et discuté les résultats de calcul dans des configurations telles que : couches limites de paroi avec ou sans gradients de pression, éventuellement en présence de la transition ou de l'aspiration, parois courbes, canaux plans ou axisymétriques à révolution, écoulements cisaillés libres, avec ou sans convection naturelle, écoulements décollés, écoulements avec rotation d'ensemble, applications hydrauliques, turbomachines, échangeurs thermiques... Le congrès EUROMECH 180 à Karlsruhe en 1984, a permis de faire le point des derniers progrès réalisés avec des modèles de type $(k, \varepsilon)[18]$.

\subsection{Modélisation au second ordre}

En soulignant qu'il existe des circonstances particulières (jet pariétal [17] [18] ou canal dissymétrique [19] [20]) où le frottement turbulent ne s'annule pas avec les gradients de vitesse moyenne, Hanjalic et Launder [21] ont remplacé la loi constitutive (5) par une équation de transport pour la corrélation $\overline{u_{i} u_{k}}$. Cette démarche a été ensuite étendue à l'ensemble des contraintes turbulentes, ce que l'on appelle la modélisation au second ordre. En effet, il est possible de déduire de façon exacte, des équations de NavierStokes, les équations de transport des contraintes de Reynolds décrivant l'évolution des corrélations d'ordre deux des fluctuations de vitesse [22]. A noter que la contraction de cette équation tensorielle permet de retrouver l'équation de l'énergie cinétique de la turbulence (9).

Comparée à l'équation de l'énergie cinétique de la turbulence, l'équation tensorielle de transport des contraintes de Reynolds s'enrichit d'un terme nouveau : la corrélation pression-déformation :

$$
\overline{p\left(\frac{\partial u_{i}}{\partial x_{i}}+\frac{\partial u_{i}}{\partial x_{i}}\right)}
$$

Ce terme n'affecte pas l'énergie cinétique de la turbulence, puisque le mouvement fluctuant est à divergence nulle, mais joue un rôle important dans les équations relatives à chaque contrainte, normale ou tangentielle. D'une façon générale, le rôle de ce terme est de rendre l'état de la turbulence plus isotrope, en répartissant de l'énergie entre les contraintes, d'où son nom de redistribution.

La modélisation de la redistribution est basée sur l'intégration d'une équation de Poisson relative à la pression fluctuante. Elle conduit à deux mécanismes distincts : i) l'un, de nature linéaire, est qualifié rapide, car il résulte de l'action du mouvement moyen lui-même (à travers les gradients de vitesse moyenne), ii) l'autre, de nature quadratique, est qualifié de lent, car il assure le retour à l'isotropie à une échelle de temps empruntée au taux de décroissance de la turbulence $(T=k / \varepsilon)$ tout en étant proportionnel au degré de l'anisotropie de l'écoule- 
ment. Ainsi, en contraste avec le modèle $(k, \varepsilon)$ qui a seulement une échelle caractéristique, la modélisation au second ordre introduit en quelque sorte deux échelles différentes, l'une empruntée du mouvement moyen, et l'autre du mouvement fluctuant. Signalons que les méthodes spectrales développées par l'E.C.L. concernent sur un nombre infini d'échelles.

Mais l'intégration de l'équation de Poisson pour la pression fluctuante conduit également à une deuxième partie qui correspond à l'intégrale effectuée sur la frontière du domaine. Cette partie, négligeable à l'intérieur du domaine d'écoulement, devient importante lorsqu'on est près des parois solides. Physiquement, elle traduit l'effet de miroir qui reflète les effets des fluctuations de pression. Concrètement, ce terme agit dans le sens opposé du terme principal de la redistribution car il cherche à restituer l'anisotropie des contraintes normales dans la zone très proche des parois. Dans les applications pratiques, ce terme n'a pas donné encore des résultats très satisfaisants, et des aménagements ont été apportés pour y remédier [23].

Dans les équations de transport des contraintes de Reynolds, les corrélations triples (également issues des termes convectifs) ont été modélisées par simplification des équations de transport des mêmes corrélations triples [22]. Pour tous les détails de méthodologie et de pratique, le lecteur est prié à se reporter à la référence [24].

Une fois modélisée, l'équation tensorielle de transport des contraintes de Reynolds s'écrit :

$$
\begin{aligned}
& \frac{\partial}{\partial t}\left(\bar{u}_{i} u_{i}\right)+\bar{U}_{k} \frac{\partial}{\partial x_{k}} \overline{u_{i} u_{i}}=P_{i j}+\alpha\left(P_{i j}-\frac{2}{3} P \delta_{i j}\right) \\
& +\beta k\left(\frac{\partial \bar{U}_{i}}{\partial x_{i}}+\frac{\partial \bar{U}_{i}}{\partial x_{i}}\right)+\gamma\left(D_{i j}-\frac{2}{3} P \delta_{i i}\right) \\
& -C_{1} \frac{\varepsilon}{k}\left(\overline{u_{i} u_{i}}-\frac{2}{3} \bar{k} \delta_{i j}\right)+\left(C_{1}^{\prime} \frac{\bar{\varepsilon}}{\bar{k}}\left(\overline{u_{i} u_{i}}-\frac{2}{3} \bar{k} \delta_{i i}\right)\right. \\
& \left.+C_{2}^{\prime}\left(P_{i j}-D_{i j}\right)\right) \cdot \frac{\overline{k^{3 / 2}}}{\varepsilon_{i n}} \\
& +\frac{\partial}{\partial x_{k}}\left[\left(C_{i} \frac{\bar{k}}{\varepsilon} \overline{u_{k} u_{1}} \frac{\partial}{\partial x_{1}} \overline{u_{i} u_{i}}\right)\right]-\frac{2}{3} \bar{\varepsilon} \delta_{i i}
\end{aligned}
$$

avec :

$$
\begin{array}{ll}
P_{i i} & =-\left(\overline{u_{i} u_{k}} \frac{\partial \bar{U}_{i}}{\partial x_{k}}+\overline{u_{i} u_{i}} \frac{\partial \bar{U}_{i}}{\partial x_{k}}\right) \\
D_{i j} & =-\left(\overline{u_{i} u_{k}} \frac{\partial \bar{U}_{k}}{\partial x_{i}}+\overline{u_{i} u_{k}} \frac{\partial \bar{U}_{k}}{\partial x_{i}}\right)
\end{array}
$$

Comme pour les modèles à deux équations, on a besoin également ici une équation décrivant l'évolution d'une échelle de longueur caractéristique de la turbulence. C'est le taux de dissipation qui assure ce rôle, et on utilise également une équation de transport analogue à (11).

Bien que la modélisation au second ordre soit développée depuis plus d'une dizaine d'années, elle n'a pas été souvent appliquée pour des écoulements complexes, là où elle trouve toute sa raison d'être. Car paradoxalement, la complexité numérique de résolution fait que la modélisa- tion au second ordre n'est appliquée qu'aux écoulements cisaillés minces, là où la simplicité (relative) des configurations a permis aux modèles à concept de viscosité turbulente d'être souvent suffisants. Ainsi, bien souvent on a l'impression que le peu d'améliorations apportées par la modélisation au second ordre ne justifie pas la lourdeur de sa mise en œuvre.

Si la modélisation au second ordre n'est pas souvent appliquée aux configurations d'écoulement complexes, ce n'est pas principalement à cause du coût élevé des calculs (car le nombre d'équations à résoudre est plus élevé) mais à cause plutôt de la structure mathématique des équations à résoudre. Nous avons souligné précédemment que l'avantage des modèles à concept de viscosité turbulente est le renforcement des termes linéaires dans les équations convection-diffusion à résoudre. Il est alors aisé de respecter des nombres de Reynolds locaux peu élevés. Cet avantage disparait ici: le nombre de Reynolds local retrouve sa valeur nominale (convection/diffusion moléculaire), les contraintes de Reynolds n'agissent que comme des termes sources, et l'équation de la quantité de mouvement a une nature de type équation d'Euler.

Or la résolution des équations d'Euler n'est pas familière aux chercheurs habitués aux écoulements isovolumes (car dans ces derniers cas, la théorie des potentiels complexes est souvent suffisante). Il faut donc se retourner vers le domaine de l'Aérodynamique des grandes vitesses où existent de nombreux systèmes numériques bien adaptés aux équations d'Euler. L'inconvénient majeur de cette démarche est que dans ces méthodes, bien souvent une viscosité artificielle a été ajoutée pour stabiliser le comportement numérique. Il est alors recommandé de bien veiller à ce que la viscosité artificielle et la viscosité numérique inhérente aux schémas ne détériorent pas le rôle de la diffusion turbulente représentée par la divergence du tenseur des contraintes de Reynolds.

\subsection{Traitements particuliers près des parois}

Tous les schémas de fermeture rencontrés jusqu'ici sont basés sur l'hypothèse des nombres de Reynolds de la turbulence élevés, ce qui signifie que l'effet de la viscosité moléculaire est supposé négligeable dans le mouvement des structures énergétiques et que le champ de déformation moyenne n'a pas d'influence sur des mécanismes de dissipation. Si cette hypothèse semble être vérifiée dans un grand nombre de l'écoulement, elle peut ne pas être valable dans des zones proches des parois solides où l'influence de la viscosité moléculaire reprend progressivement sa prédominance. Il existe alors deux démarches possibles:

i) En supposant alors que la zone affectée par la viscosité moléculaire est très mince, on peut considérer que l'écoulement dans cette partie est quasi-parallèle, ce qui revient à négliger les effets convectifs devant les effets diffusifs et les effets de sources. Dès lors, en absence des non-linéarités convectives, il est possible d'intégrer transversalement les équations aux dérivées partielles, les transformant en relations algébriques globales. Les grandeurs physiques de l'écoulement, convenablement nor- 
malisées par des valeurs pariétales (par exemple la vitesse de frottement) sont devenues des fonctions d'une seule variable : le nombre de Reynolds représentant la distance par rapport à la paroi. On obtient alors les lois de paroi qui fournissent des conditions aux limites de raccordement sur une paroi fictive où la vitesse n'est pas nulle. Cette démarche est économique, car elles ne nécessite pas un maillage très fin près des parois. Elle convient donc pour des configurations où la complexité exige déjà un maillage très important à l'intérieur du domaine et qu'un reserrement correct des mailles près des parois conduirait alors à un nombre de nœuds de calcul trop élevé. L'utilisation des lois de parois n'est satisfaisante que sous certaines conditions très strictes : équilibre productiondissipation, variation lente de la vitesse de frottement, gradient longitudinal de pression quasi nul... et surtout lorsque le raccordement a lieu à l'intérieur de la zone à dominance visqueuse. Dans des applications industrielles, il s'agit là de la seule démarche raisonnable, aussi bien en encombrement de mémoires des calculateurs qu'en coût de calcul. Nous préciserons ultérieurement certaines des améliorations à apporter à ces lois de paroi, pour étendre leurs domaines d'application.

ii) En renonçant aux lois analytiques précédentes, on peut effectuer des calculs jusqu'à la vraie paroi. On introduit alors des techniques dites de traitements à faibles nombres de Reynolds qui consistent à utiliser des lois d'amortissement pour détruire progressivement l'effet de la turbulence lorsqu'on se rapproche de la paroi. En pratique, ces traitements sont exprimés sous forme de fonctions de pondération qui varient en fonction du nombre de Reynolds de la turbulence qui représente alors le rapport entre la diffusivité turbulente et la diffusivité moléculaire.

En principe, le calcul jusqu'à la vraie paroi est une démarche plus saine. En pratique, cette approche rencontre encore des difficultés: connaissance insuffisante des grandeurs turbulentes (par exemple le taux de dissipation ou l'anisotropie turbulente) dans la sous couche visqueuse, surtout lorsque d'autres phénomène se mêlent : forts gradients de pression, instationnarité, densité variable, fortes courbures... Par ailleurs, les gradients très importants des grandeurs turbulentes dans cette zone pariétale nécessitent un maillage de calcul très fin, donc un coût de calcul très élevé. Enfin, la rareté des résultats expérimentaux dans ces zones, et dans les différentes situations citées précédemment, ne permet pas une détermination précise des constantes supposées universelles du modèle.

A l'heure actuelle, des améliorations sont encore à réaliser avant que cette approche puisse être considérée comme opérationnelle dans des codes industriels.

\section{Perspectives de la modélisation}

Malgré des difficultés rencontrées dans le domaine de la modélisation, ces dernières ont été relativement bien identifiées et de nombreuses études entreprises actuellement pour les surmonter, et cela avec l'aide de codes de calcul de plus en plus performants. On peut se permettre de réver au vu d'un récent rapport du National Research Council des Etats-Unis annonçant la possibilité du calcul de l'écoulement turbulent instationnaire et tridimensionnel autour d'un avion complet, en l'an 2000 !

Pour cela, les trois approches citées au début de cet exposé pourraient évoluer de la façon suivante:

- La simulation directe des écoulements turbulents continuera à fournir des résultats expérimentaux d’origine numérique, qui seront très précieux pour le perfectionnement des modèles statistiques ou semi-statistiques. Dans les dix années à venir, cette voie serait étendue à des écoulements de plus en plus complexes, mais encore de type laboratoire. L'application dans le domaine industriel resterait encore assez exceptionnelle.

- Le calcul des macro-structures et modélisation de sous-maille iraient vers des modélisations de plus en plus complètes. De ce fait, il empiéterait de plus en plus sur le domaine de la modélisation statistique et il ferait alors la jonction avec une démarche que nous avons adopté depuis quelques années à l'Institut de Mécanique des Fluides de Toulouse, démarche que nous qualifions semi-déterministe, car elle est basée sur un calcul des structures déterministes par résolution des équations de Reynolds gouvernant les grandeurs exprimées en moyennes de phase, couplées avec un modèle de turbulence prenant en compte l'effet des structures non-déterministes.

En tenant compte des moyens de calcul disponibles actuellement et dans un avenir proche, notre avis est que ce type d'approche est vraisemblablement une démarche pour résoudre, dans les dix ans à venir, des problèmes industriels, c'est-à-dire des écoulements en configurations complexes et en situations réelles.

En attendant, un certain nombre de problèmes doivent être résolus, nous citons ici certains d'entre eux, la liste n'étant pas exhaustive :

- Problème de modélisation de la turbulence en écoulements instationnaires, lorsque l'équilibre spectral de Kolmogorov est rompu.

- Problème des écoulements turbulents à densité variable, quelle que soit l'origine de cette propriété

- Problème des écoulements turbulents à distorsions rapides, à fortes courbures, à fortes rotations d'ensemble... - Problème de l'anisotropie de la turbulence, près des parois, ou à travers des ondes de choc...

- problème de modélisation dans des zones très proches des parois solides (calcul jusqu'à la paroi ou loi de paroi ?). J. Cousteix donnera d'autres détails dans le cadre de ce séminaire.

- Problème de complexité géométrique des domaines de l'écoulement: D. Jeandel présentera les aspects numériques liés à ce volet.

- Problème des frontières libres et intermittentes dans des écoulements instationnaires: M. Lesieur exposera les acquis les plus récents dans la simulation numérique directe des écoulements libres. 


\section{Références bibliographiques}

[1] Kline S.J., Morkovin M.V., Sovran G., Cockrell D.J. (1968). - "Computational of Turbulent Boundary Layers 1968 - AFOSR-IFP-STANFORD Conference". Vol. I and II., Stanford University, California, USA 94305.

[2] Kline S.J., CANTwell B.J., Lilley G.M. (1982). - "The 1980-1981 AFOSR-HTTM-STANFORD Conference on Complex Turbulent Flows: Comparison of Computation and Experiment", Vol. I, II and HI, Stanford University, 1982.

[3] BRAZA M. (1986). - "Analyse physique du comporter:ent dynamique d'un écoulement externe, décollé, instationnaire, en transition laminaire-turbulente. Application : Cylindre circulaire". Thèse de Doctorat ès Sciences, INPT, décembre 1986.

[4] Spalart P.J. (1986). - "Direct Simulation of a Turbulent Boundary Layer up to $R=1410$ ". NASA TM 89407 , décember 1986.

[5] Spalart P.J., (1986). - "Numerical Study of Sink Flow Boundary Layers", J.F.M., 172, pp. 307-328.

[6] Moser R.D., Moin P. (1984). - "Direct Numerical Simulation of Curved Turbulent Channel Flow", NASA TM. 85974.

[7] MoIN P., KıM J. (1982). - "Numerical Investigation of Turbulent Channel Flow", J.F.M. 118, pp. 341-377.

[8] D'humieres D., Lallemand P., Pommeal Y. (1986). "Simulation d"écoulements compressibles à l'aide d'un gaz sur réseau", Colloque DRET-ONERA Poitiers, Mars 1986.

[9] PRAndtL L. (1945). - "Uber ein neues Formelsystem fïr die ausgebildeten Turbulenz". Nachrichten von der Akad. der Wissenschaft in Göttingen.

[10] Cebeci T., SMith A.M.O., Mosinskis G.J. (1970). - "Solution of the incompressible turbulent boundary layer equations with heat transfer", J. Heat Transfer, 92, p. 499.

[11] Balwin B.S., Lomax H. (1978). - "Thin Layer Approximation and Algebraic Model for Separated Turbulent Flows", AIAA Paper 78-257, Huntsville, Alabama.

[12] RotTA J. (1971). - "Recent attemps to develop a generally applicable calculation method for turbulent shear flow layers". Proceedings of AGARD Conference on Turbulen Shear Flows, London.

[13] Jones W.P., LAUNDER B.E. (1972). - "The prediction of laminarisation with a two-equation model of turbulence", Int. Jl. Heat Mass Transf., No.5, pp. 301-314.
[14] Ha Minh H., Rubesin M.W. Vandromme D.D. Viegas J R. (1985). - "On the Use of Second Order Closure Modelling for the Prediction of Turbulence Boundary Layer/Shock Wave Interaction: Physical and Numerical Aspects", Inter Compuational Fluid Dymamics Sym., Tokyo, sept. 1985.

[15] Rodi W. (1984). - "Examples of Turbulence-model applications". Turbulence models and their applications. CEA-EDF-INRIA, Ecole d'été d'analyse numérique. Eyrolles 1984.

[16] RodI W. (1986). - "Turbulence Modelling for Incompressible Flows", $P C H$, Vol. 7, No. 5/6, pp. 297-324.

[17] Mathieu J. (1961). - "Contribution à l'étude aérothermique d'un jet plan évoluant en présence d'une paroi". P.S.T. du Ministère de l'Air, No. 374.

[18] Beguier C. (1971). - "Eude du jet plan dissymétrique en régime turbulent incompressible", Thèse de Doctorat d'Etat, Université de Provence. IMST.

[19] Eskinazi S., Yeh H. (1956). - "An investigation on fully developed turbulent flows in a curved channel", $J r$. Aero. Sc., Vol. 23, No.1, pp 23-25.

[20] Hanjalic K., Launder B.E. (1972). - "Fully developed asymetric flow in a plane channel", J.F.M., No. 51, p. 301.

[21] Hanjalic K., Launder B.E. (1972). - "A Reynolds stress model of turbulence and its application to thin shear flows". J.F.M., No. 52, part 4, pp. 609-638.

[22] Launder B.E., Reece G.J., Rodi W. (1975). - "Progress in the Development of a Reynolds stress turbulence closure", J.F.M., Vol. 68-3, pp. 537-566.

[23] Vandromme D.D., Ha Minh H., Viegas J., Rubesin M.W., Kollmann W. (1983). - "Second Order Closure for the Calculation of Compressible wall bounded flows with an Implicit Navier-Stokes solver", Fourth T.S.F., Karlsruhe, sept. 1983.

[24] Launder B.E. (1984). - "Second moment Closure: Methodology and Practice". Turbulence models and their applications. CEA-EDF-INRIA Ecole d'été d'analyse numérique. Eyrolles 1984

Adresse de l'auteur

Monsieur Ha Minh Hieu.

Professeur à l'Institut national polytechnique de Toulouse,

Institut de mécanique des fluides.

Avenue du Pr. Camille Soula.

31004 Toulouse. 